\title{
MODElling, CONCEPTION AND Simulation OF A DigITAL WATERMARKING SYSTEM BASED ON HYPERBOLIC GEOMETRY
}

\author{
Coulibaly Cheick Yacouba Rachid and Tiendrebeogo B. Telesphore \\ Department of Mathematics and Computer Science, Nazi Boni University, Bobo- \\ Dioulasso, Burkina Faso
}

\begin{abstract}
The digital revolution has increased the production and exchange of high-value documents between institutions, businesses and the general public. In order to secure these exchanges, it is essential to guarantee the authenticity, integrity and ownership of these documents. Digital watermarking is a possible solution to this challenge as it has already been used for copyright protection, source tracking and video authentication. It also provides integrity protection, which is useful for many types of documents (official documents, medical images). In this paper, we propose a new watermarking solution applicable to images and based on the hyperbolic geometry. Our new solution is based on existing work in the field of digital watermarking.
\end{abstract}

\section{KEYWORDS}

Digital watermarking, Harris point of interest, Poincaré disk, hyper-catadioptric projection, stereographic projection

\section{INTRODUCTION}

Securing digital documents and intellectual property is a real challenge in this era of increasing file transfer over the Internet [1].These data are easily hacked and given their value it is really important to have solutions to protect them. These solutions must protect against interception, duplication, falsification and corruption. One of these solutions is digital watermarking. Digital watermarking consists in inserting in a digital document a visible or invisible mark, containing information resistant to attacks trying to modify the mark [2]. There are several algorithms that try to find a compromise between robustness and invisibility. However, none of them fully satisfy both properties. They are limited and are intended to be used on very specific documents. To date, there is no universal model that can be adapted to any type of document. Hyperbolic geometry and its associates such as hypercatadiotry [3, p. 2012] and the Poincaré disk have many properties that we exploit in this work. Our objective is to propose an image watermarking technique that will complement existing cryptographic techniques for image delivery. In addition, we use new image processing techniques [4] hat allow us to obtain more robustness and imperceptibility. Our paper is structured as follows: we first present related work on digital watermarking using geometric techniques. We then give an overview of the principles and applications of digital watermarking, and describe our model. We then discuss some notions of hyperbolic geometry, explain the Poincare disk model, and present the principles of cryptography in the context of hyperbolic tilings. Finally, we present our digital watermarking technique, and provide a formal analysis of the robustness and perceptuality of our solution. 


\section{RELATED WORK}

The increase in digital exchanges has favored the illicit exploitation of the contents of digital documents. To overcome this problem, researchers have found solutions that involve digital watermarking. The common principle of the various solutions consists in marking the work or the object to be protected with an imperceptible signature which allows the identification of the owner while remaining resistant to the attacks.

The different watermarking systems proposed in the literature are classified in one of the three categories corresponding to their field of application which are: the spatial domain [5],the frequency domain [5] and those which act in a hybrid domain [6]. the systems that operate directly on the image are generally vulnerable to attacks, especially geometric ones, even if they are simple to set up. For digital watermarking, they use pairs or blocks of pixels to which they slightly modify and which respect previously established watermarking rules. Any geometric transformation on the image mark often makes extraction impossible. Methods that operate in a transformed plane (DCT, wavelets, Fourier, etc.) allow us to build watermarking systems that are robust against attacks of a geometric nature (rotation, windowing, etc.), or signal processing (compression, filtering, etc.).However, spatial techniques remain by far the fastest and simplest modes of attack compared to techniques that operate in the transform plane[7]. Compared to the use of cryptography to secure the exchanges, the digital watermarking offers to the stored documents or in transit an authentication and a persistent integrity. Image watermarking can therefore be a solution to secure image transfer. The objective of watermarking is to insert information into the image in an invisible and indelible way. The insertion of the message can be done in the spatial or frequency domain, or in a combination of the two domains [8] [9, p. 17]. Several existing techniques insert marks into various spatial primitives, such as the distance between a vertex and a mesh center of gravity [10, p. 16], the local triangulation density [11, p. $12]$, or the relative position of a vertex with respect to its neighbors [12, p. 15] with the exception of methods based on statistical shape descriptors [13, p. 16], these techniques are vulnerable to attacks on connectivity. To address this vulnerability, some algorithms propose to do a resampling preprocessing on the mesh to be tested before extraction [14, p. 08]. Another class of algorithms consists of decomposing the mesh in the spectral domain, and then inserting the mark into various frequency components depending on the intended application [15, p. 15] [16, p. 15].

These methods generally offer better inaudibility and also better robustness to geometric attacks. Nevertheless, the fragility against connectivity attacks still exists. For example, Laplacian spectral analysis of a 3D mesh is sensitive to changes in connectivity [15, p. 15]; iterative edge decimation to construct a multi-resolution representation also depends on connectivity [17, $\mathrm{p}$. 15]; furthermore, most wavelet analysis tools for a $3 \mathrm{D}$ mesh require that the mesh to be decomposed be at least semi regular [18, p. 15]. In this article, we propose a new digital watermarking technique that allows the creation of an imperceptible and attack-resistant signature. Our technique is based on the projective geometric transformation of the image in hyperbolic space after the extraction of the points of interest that will be used for the marking. The identification of the interest points is similar to the principle of Harris [19].

\section{WATERMARKING: TECHNIQUES AND PRINCIPLES}

For better comprehension, we introduce watermarking techniques and then provide background information on the principles of watermarking for the proposed approach, and we will present some of its applications. We start our work by mentioning some of its applications, specifically the securing of documents. We focus on discussing the principles of cryptography in light of the purpose of watermarking. 
Signal \& Image Processing: An International Journal (SIPIJ) Vol.12, No.4, August 2021

\subsection{Watermarking for Document Security}

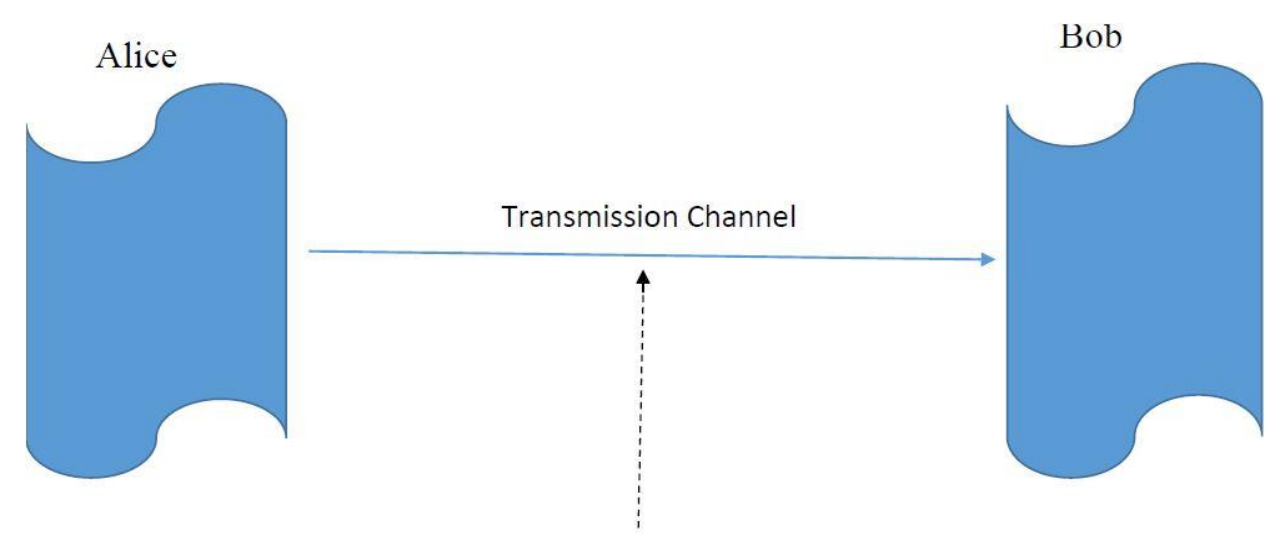

Charlie

Figure 1: General representation of communication[20]

Consider [20] the illustration in Figure 1 where a sender named Alice transfers a document to a receiver, named Bob. During this transmission, a third party, Charlie, may attempt to extract or modify the data in the document. The document can thus be intercepted and its integrity can be attacked. Figure 2 is a diagram representing key terminology used in document security, where the primary term is cryptology, which is the science of information protection. Within cryptology, the three main methods used are cryptography, steganography and watermarking. These methods differ in their algorithms, the way they manipulate information, and the length of time the information is protected. For example, the Figure 3 illustrates a weakness of processes that use cryptography but a document is vulnerable to attack after decryption. However, we can notice some points of similarity between these three methods as shown in Figure 4.

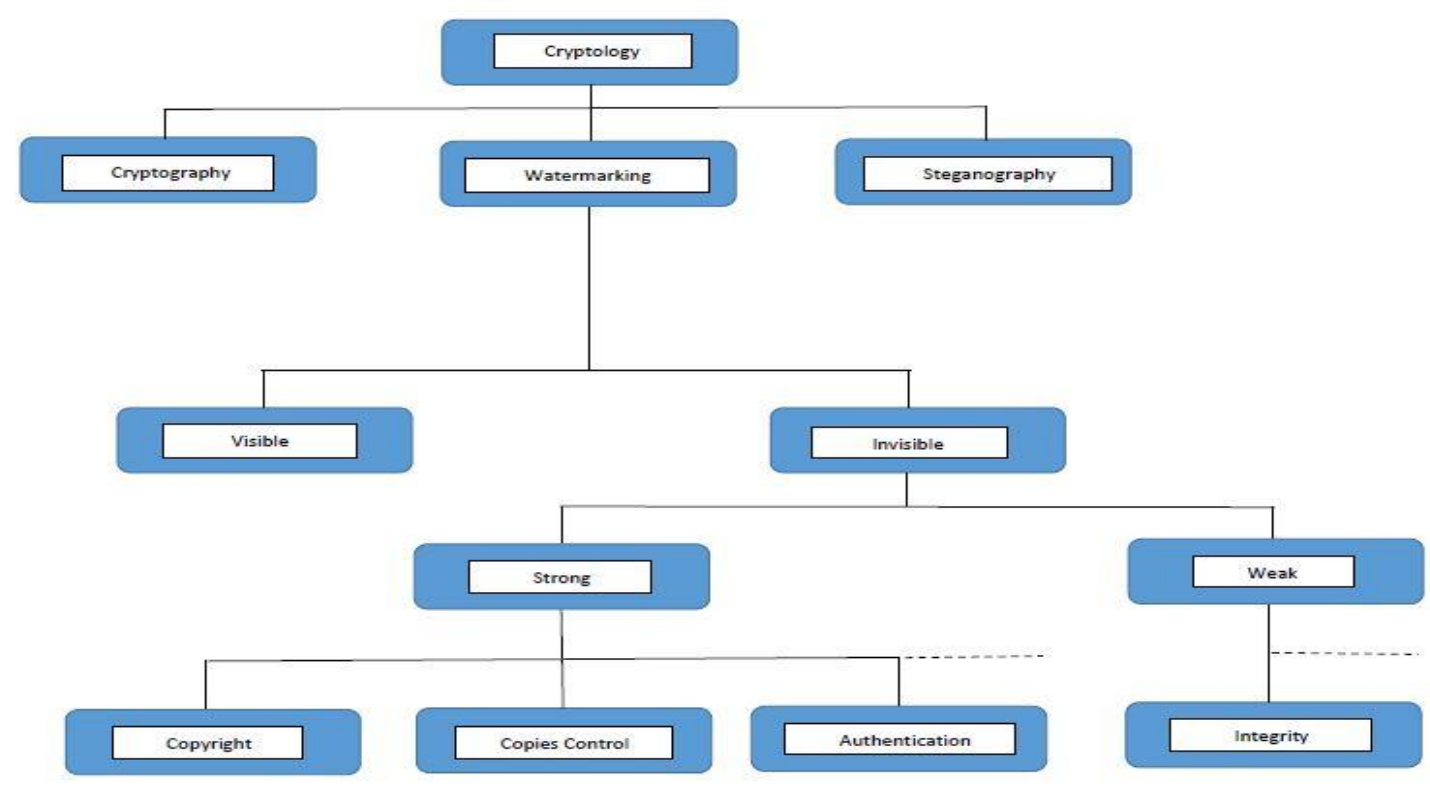

Figure 2: Security and safety methods diagram[20] 
Signal \& Image Processing: An International Journal (SIPIJ) Vol.12, No.4, August 2021

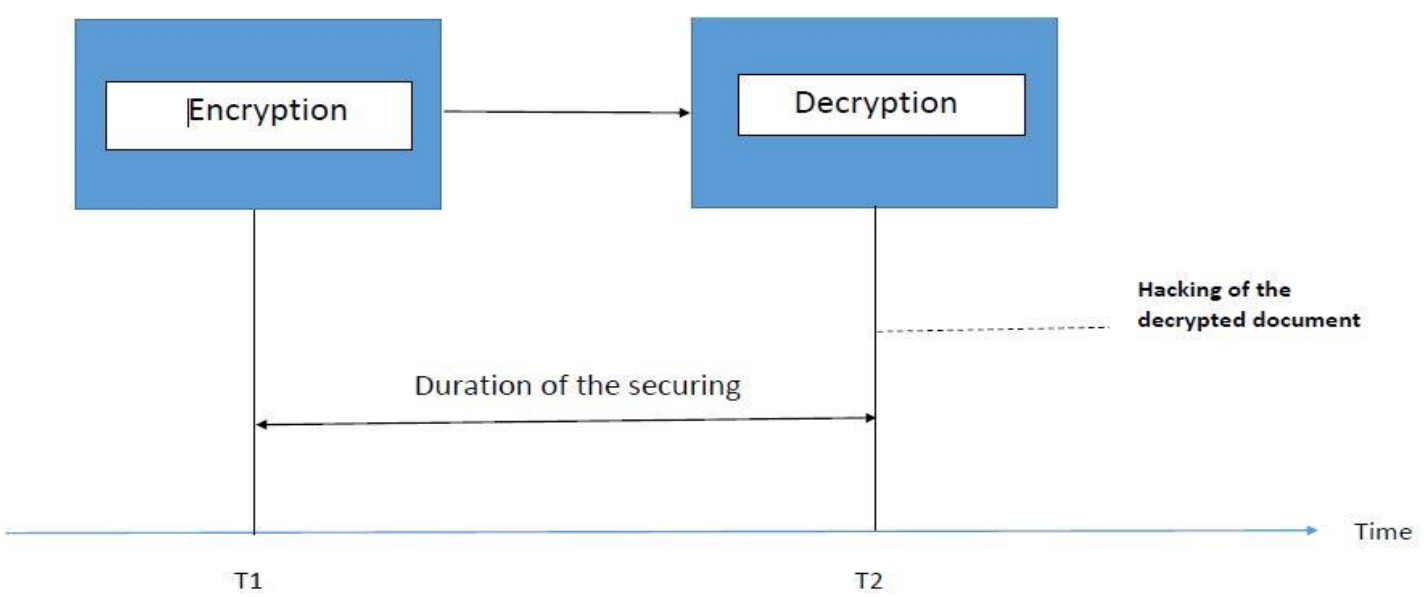

Figure 3: Encrypted document safety duration[20]

In the following, we will focus on digital documents. In general, cryptography is used to protect the information of a document during its transmission on a network. It makes the document unreadable from the moment it is encrypted until its final decryption. This is possible thanks to the use of keys. Only the owners of the key(s) have access to the encrypted information.

This method has proven to be effective in protecting information during its transmission between authorized parties. It is commonly used to prevent the viewing of certain programs on private television channels by non-paying customers.

The principle of cryptography is to scramble data for security purposes during encryption and decryption. To protect information "permanently" (i.e., beyond the transmission period), steganography is often used.

Steganography is defined as the art of hiding information in a medium. There are two approaches to steganography:

The first approach is to hide the information to be protected inside another document. This is called "information hiding" or "data hiding". The principle of this approach is similar to that of cryptography: information is inserted or extracted from the medium by means of codes generated by keys. However, unlike cryptography, the protected information is not visible in the document.

The second approach to steganography is to embed a signature in a document to protect the document from being compromised. This approach is known as "watermarking". Our work focuses on this approach to steganography. Figure 4 provides a comparative illustration of the features of watermarking versus other document security techniques. 
Signal \& Image Processing: An International Journal (SIPIJ) Vol.12, No.4, August 2021

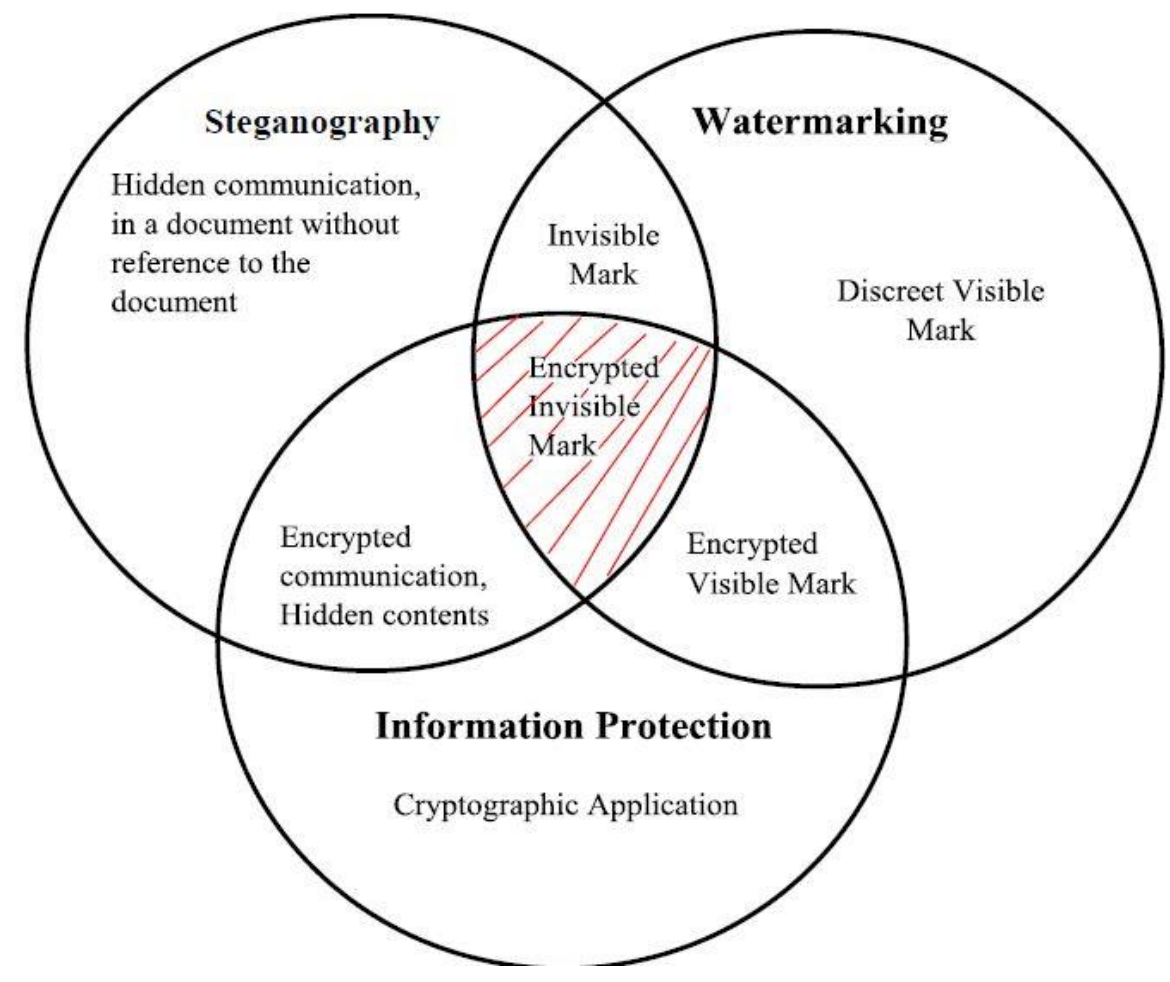

Figure 4: Relations with other safety domain

Watermarking techniques can modify documents in two different approaches. The semantic watermarking modifies the content of the document. The syntactic watermarking modifies the document storage format. In both cases, the changes applied to the document are not intended to affect its usability. On the other hand, the attacks on the documents aim at removing or modifying the mark left by the watermarking algorithm. Indeed, according to the mode of attack, the quality of the mark can be reduced (case of strong algorithms) or can be significantly modified (case of weak algorithms). In addition, in information technology there are multiple applications of watermarking, such as guaranteeing the integrity of video streams, identifying the owner, proof of ownership, tracking transactions, authentication, copy control, insertion of metadata.

\section{Principles of Watermarking}

We consider the following notations in the description of the various stages of the watermarking process: $D$ for any document and $D_{0}$ for the original document. Any digital document is defined as a series of binaries:

$D \in\{0,1\}$.A document is said to be of good quality during a transmission if and only if:

$$
F_{q}:\{0,1\}^{*} \times\{0,1\}^{*} \rightarrow\{0,1\}
$$

Where

$F_{q}$ is a quality function. 
Signal \& Image Processing: An International Journal (SIPIJ) Vol.12, No.4, August 2021

Thus, two documents are of equal quality if and only if :

$$
F_{q}\left(D, D^{\prime}\right)=1
$$

$F_{q}$ can be considered as criteria of psychological perception.

As for the watermarking model, let's consider the following model:

Consider $M$ and $D$ as a pair of algorithms, denoted by : $(D, M)$, with $M$ as the marking algorithm and $D$ as the document algorithm.

The document watermarking consists of the following:

$$
F_{M}:\{1,0\}^{*} \times\{0,1\}^{*} \rightarrow\{0,1\}^{*}
$$

Where $F_{M}$ is the watermarking function associated with

$$
M(D, I)=D^{\prime}
$$

Where $D^{\prime}$ is the watermarked document and $I$ is the information inserted in the original document (called additional information). In the remainder of this paper, we focus on documents representing digital images.

The syntactic model (consisting of insertion by addition), also referred to as the additive scheme in watermarking, is illustrated in Figure 5: a signature is added to a digital image. We study the case where the signature is generated in a pseudo-random way, on the basis of a key $K$. The signature is then transformed into a message before being added to the coefficients of the image. The coefficients of the image arise from the image itself or are the result of a space representation change.

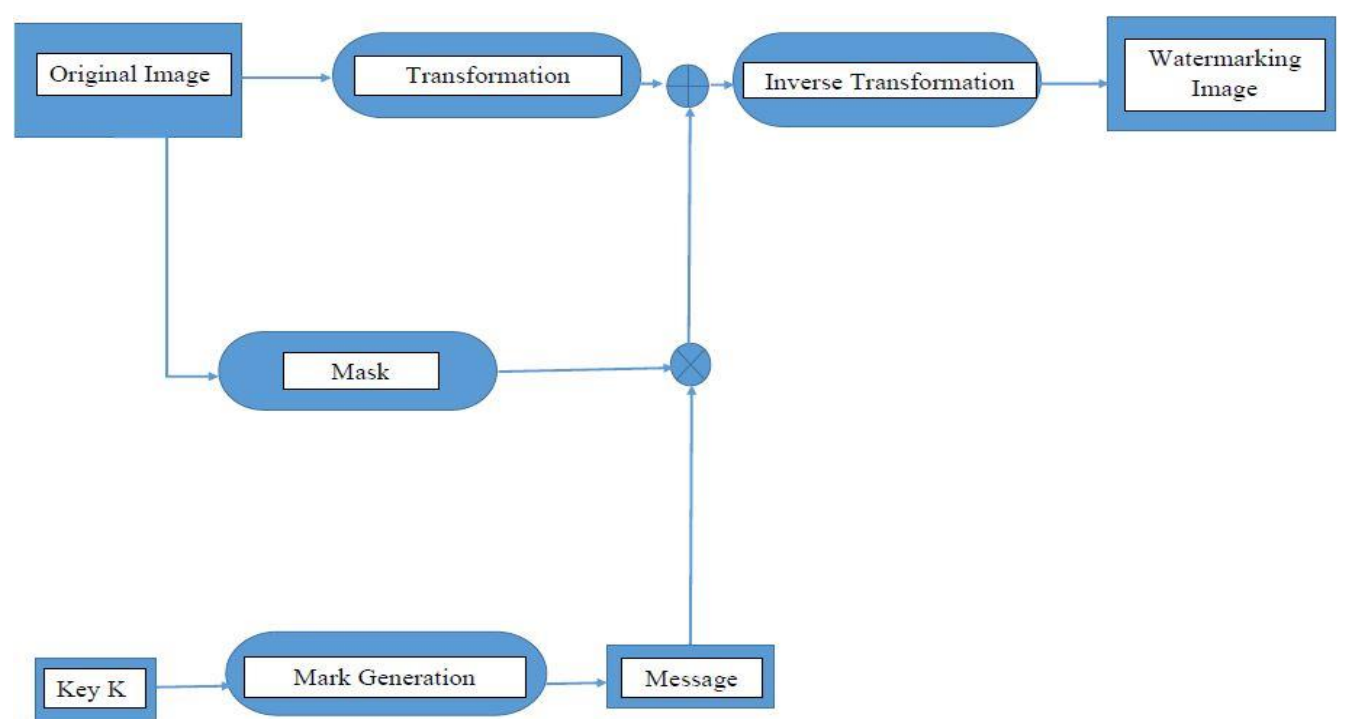

Figure 5:The additive Scheme for the signature insertion. 
he semantic model (consisting of insertion by substitution), also referred as the substitute scheme of watermarking, is illustrated in Figure 6: certain components of the image are replaced by the signature. Such a signature is then initially obtained by applying a constraint (for example a relation of order, a criterion of similarity, a geometrical property of the image) to the components according to the message to be inserted.

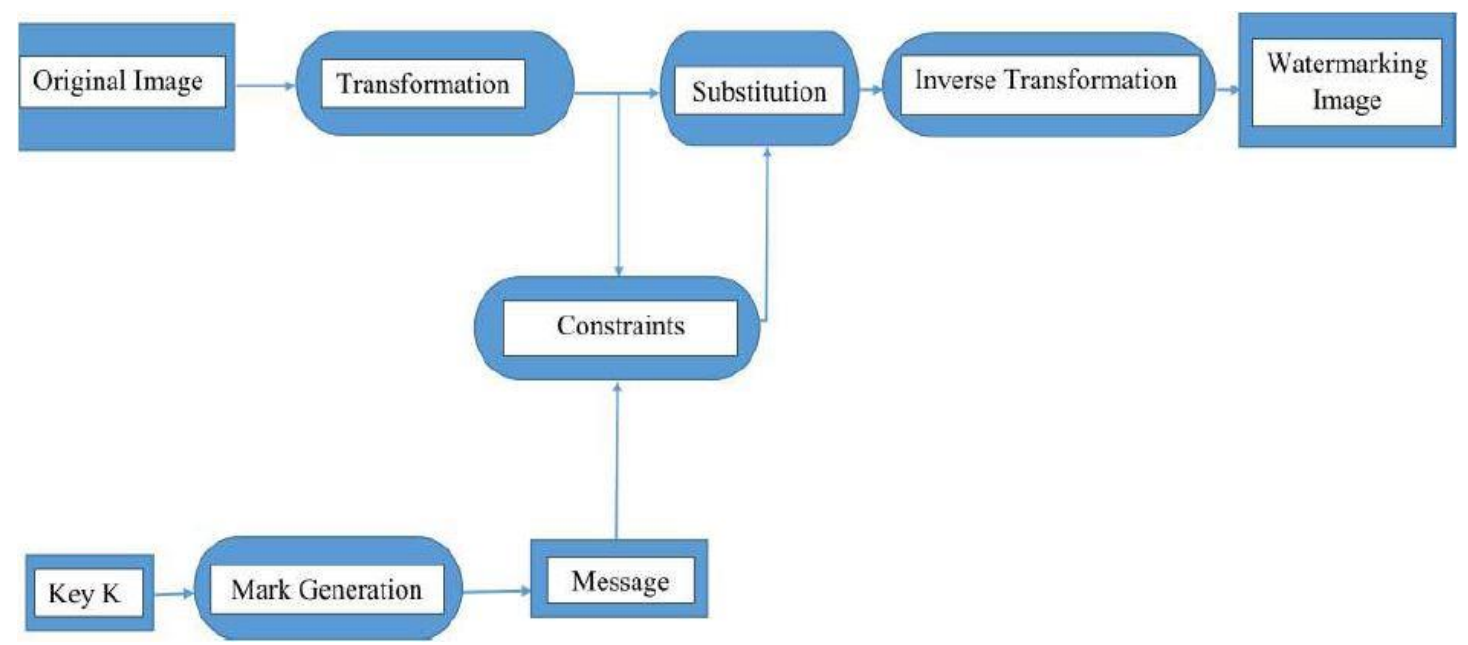

Figure 6: Substitute Scheme of the signature insertion.

With regards to watermarking detection, let us consider the following model:

Let $F_{D}$ be the detection function that allows for the recognition of the original image. This function is defined as follows:

$$
F_{D}=\{0,1\}^{*} \times\{0,1\}^{*} \rightarrow\{0,1\}
$$

Let $D^{\prime}$ and $I^{\prime}$ respectively be a suspicious document and any other (supposedly additional) information, then we can state that a document is authentic (original document), if and only if:

$$
F_{D}\left(D^{\prime}, I^{\prime}\right)=1
$$

where $I^{\prime}=I$ or $I^{\prime} \neq I$. For the sender or the addressee, $I$ will always be equal to $I^{\prime}\left(I=I^{\prime}\right)$ Properties1:

- $\forall\{D, I\}, F_{D}\left(F_{M}(D, I), I\right)=1$. These properties (in total) are called “Adjustment".

- $\forall\{D, I\}, F_{q}\left(D, F_{M}(D, I)\right)=1$. These properties (in total) are called "Inaudibility".

Figure 7 illustrates the specific case of a simplified detection technique that will be referred to in this paper in the axis of the substitution scheme. In practice, we intend to use a hybrid technique, which involves substitution and addition at the same time. However, with detection based on an additive scheme and when it is about blind technique, we could use statistical methods (where the original image is not available for detection). In this case, a measure of correlation can be made for example. 
Signal \& Image Processing: An International Journal (SIPIJ) Vol.12, No.4, August 2021

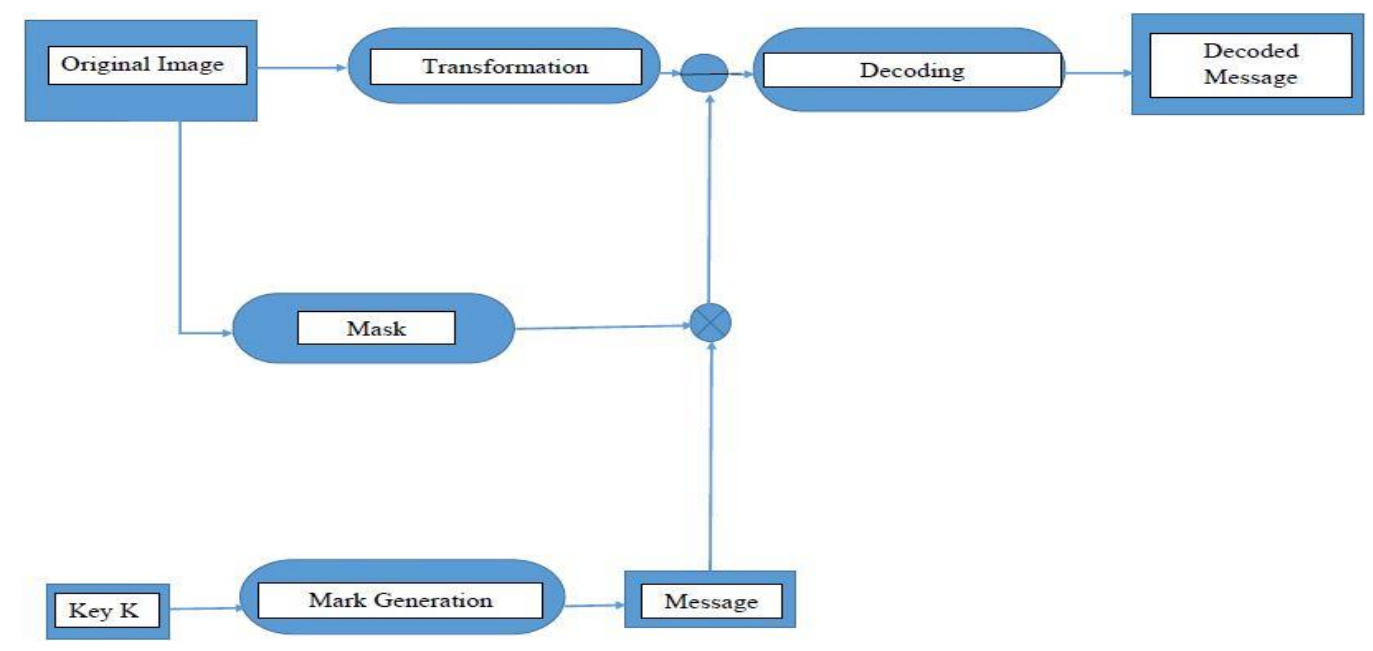

Figure 7:Detection and reading of the signature

\section{HARris Point of InTERest}

The Harris point detector is a common technique to detect points of interest in an image.

The points of interest have been described in order to reduce the maximum of information of an image in a few points.

The point of interest is found thanks to the Moravecdetector which uses the autocorrelation function of the image to measure the intensity differences between a patch and windows shifted in several directions.

The article [21] explains in more detail the points of interest of Harris.

\section{Hyperbolic GeOMETry Models}

In this section, we first present the properties of hyperbolic geometry, and then we illustrate the steps of hyperbolic tiling construction, which are essential for the creation of our mark.

\subsection{Properties of Hyperbolic Geometry}

The basis of hyperbolic geometric is driven by Euclid's five postulates[22, p. 50]. Thus, hyperbolic geometry must be understood in light of Euclidian geometry. The basic elements of Euclidian geometry are: the point, the straight line, the right angle, the intersection and the congruence among angles. The five postulates are then as follows:

The straight-line postulate: a straight-line segment can be drawn joining any two points;

The continuation postulate: any straight-line segment can be extended indefinitely in a straight line;

The circle postulate: given any straight-line segment, a circle can be drawn having this segment as radius and one of its end-points as center; 
The equal right angles postulate: all right angles are equal;

The parallel postulate: if two lines are drawn which intersect a third in such a way that the sum of the inner angles on one side is less than two right angles, then the two lines inevitably must intersect each other on that side if extended far enough.

Hyperbolic geometry, also referred to as Lobachevsky geometry, disagrees with Euclid's fifth postulate and bases itself on the findings of Legendre[23, p. 896].Our work focuses on the hyperbolic space around the dimension $n \geq 2$. The hyperbolic plan being of course the hyperbolic space of dimension (i.e., size) 2. The hyperbolic space is a homeomorphous metric space (E, d) in the Euclidean space $\mathbf{R}^{n}$.This is interesting because of the size of its group of isometries, which is of dimension $n(n+1) / 2$; only the Euclidean and spheric spaces are as good. Although there are many models of this metric space[24, p. 04], we are particularly interested in the model of the half-hyperboloid $\left(\mathrm{H}^{n}, d\right)$.
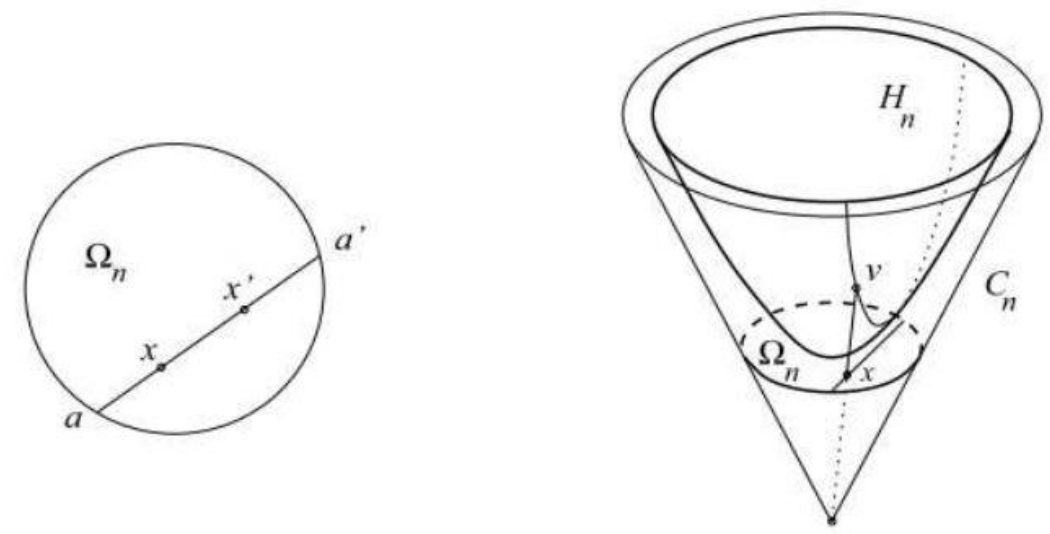

Figure 8: Hyperboloide

Figure 8 illustrates our hyperbolic geometric model where angles and geodesic are deformed. It remains nonetheless useful in describing the isometries of the hyperbolic space. This representation also plays a crucial role in the construction of tilings in following sections. Let us note $q$ the Lorentzian quadratic form on the vectorial space $V=\mathbf{R}^{n}$ and $b$ the associated bilinear form. For $v=\left(x_{0}, x_{n}\right)$ and $w=\left(y_{0}, y_{n}\right)$ we have: $b(v, w)=-x_{0} y_{0}+x_{1} y_{1}+\ldots+x_{n} y_{n}$ and $q(v)=b(v, v)$.

We note $C_{n}$ as the cone of the future of this Lorentzian form and $H_{n}$ as the top of the hyperboloid:

$$
\begin{aligned}
& C_{n}=v \in \mathbf{R}^{n+1} / q(v)<\operatorname{Oand}_{\mathrm{O}}>\mathrm{O} \\
& \boldsymbol{H}_{n}=v \in \mathbf{R}^{n+1} / q(v)=-1 \text { and } x_{\mathrm{o}}>\mathrm{O}
\end{aligned}
$$

We still define the distance of two points $v$ and $v^{\prime}$ of $H_{n}$ as: 


$$
d\left(v, v^{\prime}\right)=\left|\log \left(\left[p, p^{\prime}, v, v^{\prime}\right]\right)\right|
$$

Where $p, p^{\prime}$ are the points of the edge of the cone $C_{n}$ which are on the same right as $v$ and $v^{\prime}$ and where $\left[\mathrm{p} ; \mathrm{p} ; \mathrm{v} ; \mathrm{v}^{\prime}\right]$ is the bi-report of these four aligned points. Given such a model of the hyperboloid, it is easy to describe the group of the isometries of the hyperbolic space. Indeed, the regular hyperbolic tiling is a part of the group of isometries. By analogy with the Euclidian geometry, we define the angle in hyperbolic geometry. We verify that, for the corresponding ball, it coincides with the Euclidian angle.

The angle between two geodesic paths $\mathrm{c} 1$ and $\mathrm{c} 2$ of the hyperbolic space, which arise from the same point $x_{0}=c_{1}(0)=c_{2}(0)$ is the real $\theta \in[0, \pi]$ defined by the formula of the rope:

$$
2 \times \sin \theta / 2=\lim _{x \rightarrow 0} \frac{1}{t} \times d\left(c_{1}(t), c_{2}(t)\right)
$$

This definition does not depend on a specific model. As each of the models are included in a Euclidean space, we can differentiate the hyperbolic angle from the Euclidean angle.

\subsection{Hyperbolic Tiling}

In this section, we begin with Poincare's construction of periodic tiling for the hyperbolic plan. This is followed by an overview of the hyperbolic analogues of the theorems of Bieberbach. We begin with the dimension 2. Finally, we present the construction of non-periodic tiling, by means of a single convex tile, for the hyperbolic plan. Regarding the construction of periodic tiling for the hyperbolic plan, let us begin by describing in full the simplest construction of periodic pavements for the hyperbolic plan as illustrated in Figure 9.
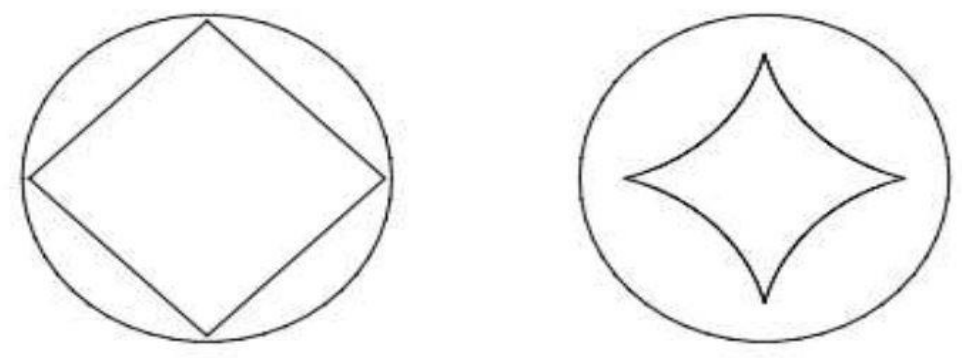

Figure 9 : A convex polygon in both projective and conform balls

As shown in Figure 10, this mechanism can infinitely tile the plan hyperbolic 


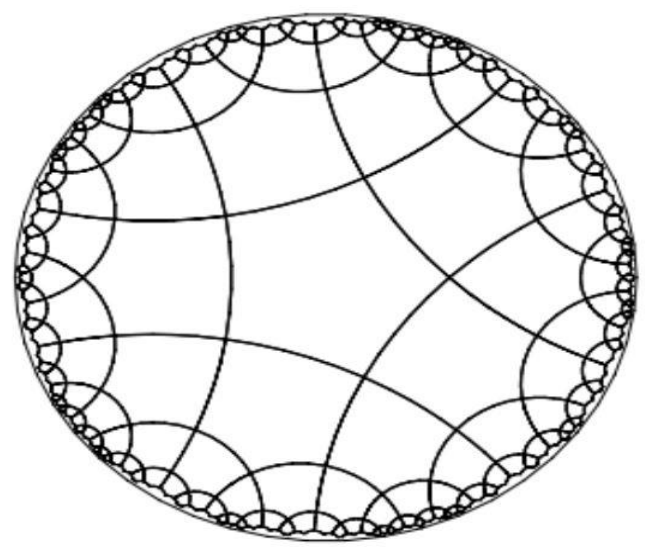

Figure 10: The hyperbolic tiling in an infinity of the regular pentagons in the projective and conform balls

\subsection{PoincaréDisk Model}

The Poincarédisk is the unit disk called D which is the whole universe. Its border consists of unit circles $\tau$ and is infinite. Points are represented by points; however, a Euclidian straight line becomes the arc of a circle, which we call geodesic. One property of the Poincare model can be misleading and that is distances are not preserved. If we observe the Poincare model from an outside perspective, distance appears smaller than in reality (i.e. inside the plane). Because the model is a representation of the hyperbolic plane in the Euclidean plane. In fact, the points which seem the closest to the unit circle are, in reality, far away from the latter. The hyperbolic plane has a boundary circle at infinity represented in the Poincare unit disk model (i.e the open unit disk) by a circle of radius 1 and centered on the origin $\mathrm{O}$. The open unit disk around $\$ \mathrm{P} \_0$ is the set of points whose complex modulus is less than 1: $|w|<1$ [25, p. 12].

$$
\text { With }|w|=\sqrt{\left(W_{\mathrm{Re}}\right)^{2}+\left(W_{\mathrm{Im}}\right)^{2}}
$$

It is important to remember certain hyperbolic tiling properties when considering Poincaré disk model.

An elementary property of Euclidean space is the impossibility of creating more than two halfplanes without their crossing. Our integration is based on the geometric property of the hyperbolic plane which allows the creation of areas called half-planes. As explained in[26], in the hyperbolic plane, we can create $\$ \mathrm{n} \$$ of pairwise disjoint half-spaces, whatever the value of $n$.

In the Poincare disk model, the distances between any two points $M$ and $N$ are given by curves minimizing the distance between these two points and are called hyperbolic plane geodesics. To calculate the length of a geodesic between two points $M$ and $N$ and thus obtain their hyperbolic distance $d_{H}$, we use the Poincaré metric which is an isometric invariant :

$$
d_{H}(M, N)=\arg \cosh (1+2 \Delta)
$$

With 
Signal \& Image Processing: An International Journal (SIPIJ) Vol.12, No.4, August 2021

$$
\Delta=\frac{|M-N|^{2}}{\left(1-|M|^{2}\right)\left(1-|N|^{2}\right)}
$$

For more details on the Poincaré metric, we refer the reader to the proof $[27$, p. 06]

\section{BaSic Principles}

\subsection{Our use of the Central Catadioptric System Geometry}

Our watermarking solution, combines hyperbolic mirrors with the Poincaré disk model and can be used in conventional cameras.It functions as a catadioptricsensor which is used by the majority of systems that are based on omnidirectional vision. The constraint on the central point called $O_{C}$ (corresponding to the second focal spot of the mirror and the optical center of the Poincaré) implies that the straight lines surrounding any point of the space belonging to the original image, which we wish to watermark along with its projection onto the hyperbolic mirror, cross a unique point on the Poincaré disk[28, p. 05].Under this constraint, every pixel on the image plan that belongs to the Poincaré disk measures the intensity of the light reflected by the optical beam onto the hyperbolic mirror passing through the central point. This is the first focus/focal point of the optical mirror (called O) in a particular direction. The coordinates of its impact point on the Poincaré disk can be determined as explained in the remainder. Such an approach is interesting as it generates a correct geometric perspective that allows for a simplification of the projection models and thus a simplification of the theoretical and practical image processing. In fact, tools developed within the context of the visions perspective are then often adapted tothe set of the central sensors. However, this condition is satisfied only for very particular reflector surfaces. Baker and Nayar in [29, p. 99] determined the class of all the central catadioptric. In this case, we are only concerned with the hyper-catadioptric system (i.e., the catadioptric system based on hyperbolic mirrors).

\subsection{Our Hyper-Catadioptric Model for Watermarking}

Let us consider our catadioptric system obtained by combining an hyperboloid mirror with a lens in the Poincare disk form of focal $f_{e}$, the optical center of which is merged with the second foyer of the hyperbolic mirror, which we called $O_{C}$.

Our approach for building the hyper-catadioptric image on the Poincaré disk is illustrated in Figure 11 


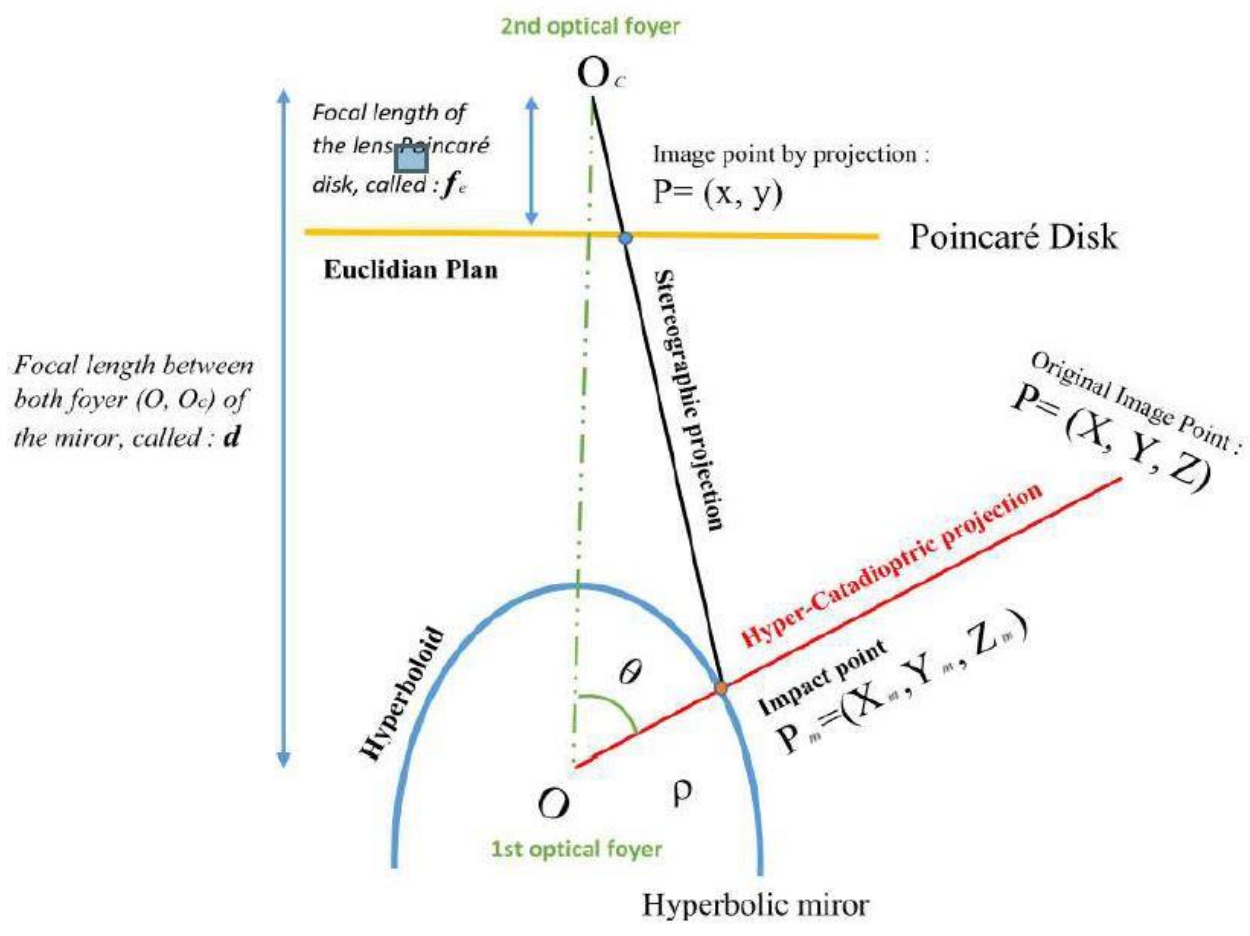

Figure 11:Building the Hyper-Catadiotric image on the Poincare disk

The approach shown in Figure 11 uses a subset of original image points that we wish to watermark, called $P(X, Y, Z)$ (considered to be the beginning of a mark element). Then, we first proceed to a hyper-catadioptric projection on the hyperbolic mirror to obtain points called $\$$ $P_{m}\left(X_{m}, Y_{m}, Z_{m}\right)$, we then make a stereographic projection onto the Poincaré disk of the various points defined by $p(x, y)$ on the mirror.

\subsection{Image Coordinates Computing}

It has been proved [30, p. 02], and we agree, that the polar equation of our hyperbolic mirror is:

$$
p=\frac{p}{1+e \times \cos (\theta)}
$$

In fact, the reduced Cartesian equation of hyperbole is equal to:

$$
\frac{X^{2}}{a^{2}}-\frac{Y^{2}}{b^{2}}
$$

Let us put $c=\sqrt{a^{2}+b^{2}}$ corresponding to focal half-length. Thus $e=\frac{c}{a}$ and $p=\frac{b^{2}}{a}$ correspond respectively to the eccentricity and parameter given in the polar equation 16 . 
Let us consider the projection case. Let the 3D point $p=(X, Y, Z)^{T}$ be projected onto the hyperbolic mirror at the point $p_{m}=\left(X_{m}, Y_{m}, Z_{m}\right)$ (hyper-catadioptric projection) where:

$$
\left(X_{m}, Y_{m}, Z_{m}\right)^{T}=\frac{p}{\sqrt{X^{2}+Y^{2}+Z^{2}}}(X, Y, Z)^{T}
$$

This point is then projected onto the image plan associated to the Poincare disk at the point $P=(z, y)^{T}$ by means of the following projection matrices:

$$
\left(\begin{array}{l}
x \\
y \\
z
\end{array}\right) \square\left(\begin{array}{ccc}
f e & 0 & 0 \\
0 & f e & 0 \\
0 & 0 & 1
\end{array}\right)\left(\begin{array}{cccc}
1 & 0 & 0 & 0 \\
0 & 1 & 0 & 0 \\
0 & 0 & 1 & d
\end{array}\right)\left(\begin{array}{l}
X_{m} \\
Y_{m} \\
Z_{m} \\
1
\end{array}\right)
$$

Where $d$ is distance between the optical foyer of the Poincare disk and the first foyer of the hyperbolic mirror.

Thus, to respect the unique point of view, $d=\frac{2 \times e \times p}{1-e^{2}}$ (the Poincare disk being situated in the hyperboloid second optical foyer) and as $\cos (\theta)=Z /|P|$, we end in the following relation:

$$
p(x, y)=\left(\frac{\frac{1-e^{2}}{1+e} f e X}{\frac{2 e}{1+e^{2}} \sqrt{X^{2}+Y^{2}+Z^{2}}+Z}, \frac{\frac{1-e^{2}}{1+e} f e Y}{\frac{2 e}{1+e^{2}} \sqrt{X^{2}+Y^{2}+Z^{2}}+Z}\right)
$$

\section{Process of the Mark's Creation}

For each point of interest of Harris we make a hyper-catadioptric projection on a hyperbolic mirror then we calculate the coordinates of their images on the hyperboloid. Then we make a stereographic projection (the half hyperboloid) in the Poincare disk then we calculate the coordinates of the different points in the Poincaré disk. The algorithm 3 describes in a way the process of creation of the mark 
Signal \& Image Processing: An International Journal (SIPIJ) Vol.12, No.4, August 2021
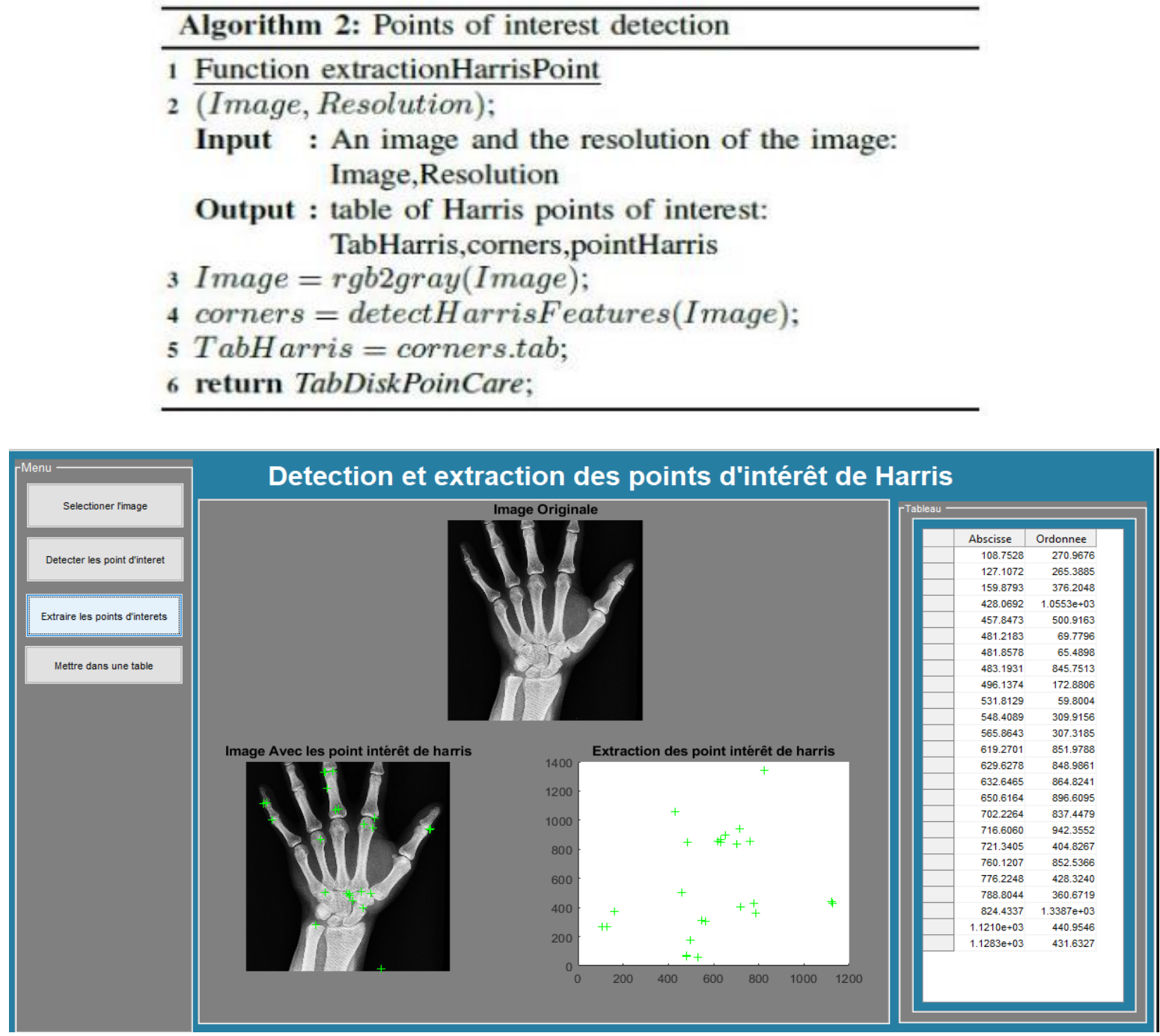

Figure 12:Detection and extraction of Harris points of interest from an image

\section{Digital Watermarking ANd Mark Extraction Process}

To do the digital watermarking, we used a hybrid technique, the Discrete Cosine Transform (DCT) combined with the Wavelet Transform (DWT).

\subsection{Digital Watermarking Process}

The watermarking by the DWT technique combined with DCT is described by the following steps[31].

Step 1: Apply DWT to decompose the cover host image into four non-overlapping multiresolution sub-bands: LL1, HL1, LH1, and HH1.

Step 2: Apply DWT again to sub-band HL1 to get four smaller sub-bands and choose the HL2 sub-band Or, apply DWT to sub-band HH1 to get four smaller and choose the HH2 sub-band

Step 3: Divide the sub-band HL2 (or HH2) into 4 x 4 blocks. 
Signal \& Image Processing: An International Journal (SIPIJ) Vol.12, No.4, August 2021

Step 4: Apply DCT to each block in the chosen sub-band (HL2 or HH2).

Step 5: Re-formulate the grey-scale watermark image into a vector of zeros and ones.

Step 6: Generate two uncorrelated pseudorandom sequences. One sequence is used to embed the watermark bit $0\left(P N_{0}\right)$ and the other sequence is sued to embed the watermark bit $1\left(P N_{1}\right)$. Number of elements in each of the two pseudorandom sequences must be equal to the number of mid-band elements of the DCT-transformed DWT sub-bands.

Step 7: Embed the two pseudorandom sequences, $\left(P N_{0}\right)$ and $\left(P N_{1}\right)$, with a gain factor $\alpha$, in the DCT transformed 4x4 blocks of the selected DWT sub-bands of the host image. Embedding is not applied to all coefficients of the DCT block, but only to the mid-band DCT coefficients. If we donate $\mathrm{X}$ as the matrix of the midband coefficients of the DCT transformed block, then embedding is done as follows:

If the watermark bit is 0 then

$X=X+\alpha^{*} P N_{0}$ otherwise

if the watermark bit is 1 then, $X=X+\alpha * P N_{1}$

Step 8: Apply inverse DCT (IDCT) to each block after its mid-band coefficients have been modified to embed the watermark bits as described in the previous step.

Step 9: Apply the inverse DWT (IDWT) on the DWT transformed image, including the modified sub-band, to produce the watermarked host image.

\subsection{Process of Mark Extraction}

the procedure of extraction of the mark is described in the following steps [31]:

Step 1: Apply DWT to decompose the watermarked image into four non-overlapping multiresolution subbands: LL1, HL1, LH1, and HH1.

Step 2: Apply DWT to HL1 to get four smaller subbands, and choose the sub-band HL2 Or, apply DWT to the HH1 sub-band to get four smaller sub-bands, and choose the HH2 sub-band.

Step 3: Divide the sub-ban HL2 (or HH2) into $4 * 4$ blocks.

Step 4: Apply DCT to each block in the chosen sub-band (HL2 or HH2), and extract the midband coefficients of each DCT transformed block.

Step 5: Regenerate the two pseudorandom sequences $P N_{0}$ and $P N_{1}$ using the same seed used in the watermark embedding procedure.

Step 6: For each block in the sub-band HL2 (or HH2), calculate the correlation between the midband coefficients and the two generated pseudorandom sequences ( $P N_{0}$ and $\left.P N_{1}\right)$. If the 
Signal \& Image Processing: An International Journal (SIPIJ) Vol.12, No.4, August 2021

correlation with the $P N_{0}$ was higher than the correlation with $P N_{1}$, then the extracted watermark bit is considered 0 , otherwise the extracted watermark is considered 1 .

Step 7: Reconstruct the watermark using the extracted watermark bits, and compute the similarity between the original and extracted watermarks.

\subsection{Performance Evaluation}

For performance evaluation we use the PSNR, NCC and IF.

- PSNR [32] is an image quality measure used to find of the quality of the cover image before and after the message is inserted. To determine the PSNR, we must first obtain the value of MSE. The higher the PSNR value, the better the quality of the watermarked image and the more similar it is to the cover image. The PSNR value can be determined using the equation below.

$$
M S E=\frac{1}{m n} \sum_{i=1}^{m} \sum_{j=1}^{n}\left(x_{i j}-y_{i j}\right)^{2}
$$

$\mathrm{m}$ number of rows in cover image

$\mathrm{n}$ number of columns in cover image

$x_{i j}$ pixel value from cover image

$y_{i j}$ pixel value from stego image

$$
\frac{10 \log _{10}[\max (\max (x), \max (y))]^{2}}{|x+y|^{2}}
$$

- NCC [32] (normalized correlation coefficient) is a measure of the reliability of the result after mark extraction. If the value of NCC is close to 1 , then the similarity is also closer to the image of the original message.

$$
N C C=\frac{\sum_{i=1}^{m} \sum_{j=1}^{n} w(i, j) x w^{\prime}(i, j)}{\sqrt{\sum_{i=1}^{m} \sum_{j=1}^{n} w(i, j)} \sqrt{\sum_{i=1}^{m} \sum_{j=1}^{n} w^{\prime}(i, j)}}
$$

Where

$$
\begin{aligned}
& w(i, j) \text { coordinates of the pixel value of the cover image } \\
& w^{\prime}(i, j) \text { coordinates of the pixel value of the mark }
\end{aligned}
$$

The Figure 13 illustrates the digital watermarking, the extraction of the marks as well as the calculation of the performance measurement. 
Signal \& Image Processing: An International Journal (SIPIJ) Vol.12, No.4, August 2021

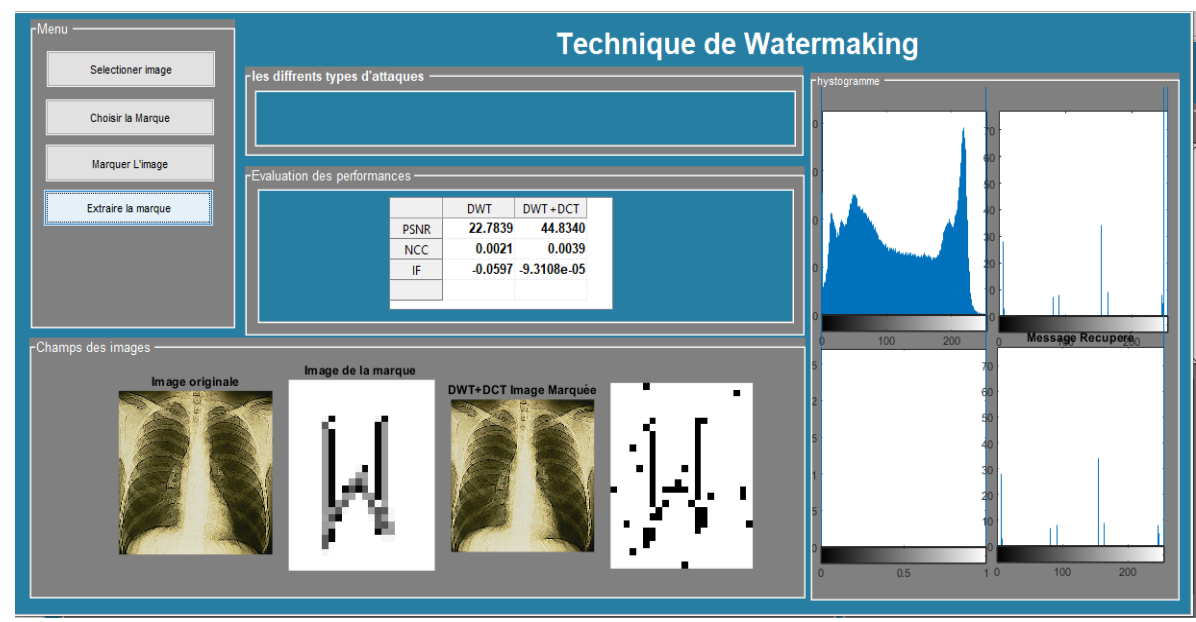

Figure 13:DWT and DCT Watermarking

\section{CONCLUSION AND FUtURE WORK}

In this paper, we have proposed a new digital watermarking technique based on the properties of projective hyperbolic geometry. Our solution is based on a hybrid watermarking technique applied to an image and integrates to this image a model resulting from the use of a hyperbolic mirror and a Poincaré disk sensor. We have formally described our model and realized it. Our future work will consist of implementing all aspects of our solution and performing a performance analysis between our solution and existing ones.

\section{REFERENCES}

[1] B. Macq, P. R. Alface, and M. Montanola, 'Applicability of Watermarking for Intellectual Property Rights Protection in a 3D Printing Scenario', in 20th International Conference on 3D Web Technology, 2015, pp. 89-95.

[2] Z. Yuefeng and L. Li, 'Digital Image Watermarking Algorithms Based on Dual Transform Domain and Self-Recovery', Int. J. Smart Sens. Intell. Syst., vol. 8, no. 1, pp. 199-219, 2015.

[3] F. Comby, C. C. de Kerleau, and O. Strauss, 'Étalonnage de camérascatadioptriqueshyperboloïdes', p. 14.

[4] M. Kaur and V. K. Attri, 'A Survey on Digital Image Watermarking and Its Techniques', Int. J. Signal Process. Image Process. Pattern Recognit., vol. 8, no. 5, pp. 145-150, 2015.

[5] Y. U., S. J.P., S. D., and S. P.K., 'Different Watermarking Techniques \& its Applications: A Review', Int. J. Sci. Eng. Res., vol. 5, no. 4, pp. 1288-1294, 2014.

[6] L. Saini and V. Shrivastava, 'Analysis of Attacks on Hybrid DWT-DCT Algorithm for Digital Image Watermarking With MATLAB', Jul. 2014.

[7] G. Chawla, R. Saini, and R. Y. Kamaldeep, 'Classification of Watermarking Based upon Various Parameters', Int. J. Comput. Appl. Inf. Technol., vol. 1, no. 2, pp. 16-19, 2012.

[8] K. D. Nagpal and D. S. Dabhade, 'Performance Comparison of Two Hybrid Techniques for Image Steganography in Frequency Domain', Int. J. Innov. Res. Comput. Commun. Eng., vol. 3, no. 6, pp. 68-74, 2015.

[9] M. Ghazvini, E. M. Hachrood, and M. Mirzadi, 'An Improved Image Watermarking Method in Frequency Domain’, J. Appl. Secur. Res., vol. 12, no. 2, pp. 260-275, 2017.

[10] Y. B. Amar, I. Trabelsi, N. Dey, and M. S. Bouhlel, 'Euclidean Distance Distortion Based Robust and Blind Mesh Watermarking', Int. J. Interact. Multimed. Artif. Intell., vol. 4, no. 2, pp. 46-51, 2016.

[11] O. Hosam, W. M. Sheta, B. A. B. Youssef, and M. A. Abdou, 'Public Watermarking Scheme for 3D Laser Scanned Archeological Models', in IEEE Symposium on Computers and Communications, 2012, pp. 382-389. 
Signal \& Image Processing: An International Journal (SIPIJ) Vol.12, No.4, August 2021

[12] L. Gil-Je and Y. Kee-Young, 'An Improved Double Image Digital Watermarking Scheme Using the Position Property', J. Multimed. Tools Appl., vol. 74, no. 17, pp. 7261-7283, 2015.

[13] B. Saliha and L. Slimane, 'Efficient Descriptor for Full and Partial Shape Matching', J. Multimed. Tools Appl., vol. 75, no. 6, pp. 2989-3011, 2016.

[14] K. Wang, G. Lavoué, F. Denis, and A. Baskurt, 'Hierarchical Watermarking of Semiregular Meshes Based on Wavelet Transform', IEEE Trans. Inf. FORENSICS Secur., vol. 3, no. 4, pp. 620-634, 2008.

[15] S. S. Sujatha, 'Blind Wavelet Based Watermarking Technique for Image Authentication', Int. J. Adv. Res. Comput. Sci., vol. 6, no. 1, pp. 127-131, 2015.

[16] Y. H. Wankhede and S. D. Mali, 'Data Hiding Technique Using Audio Watermarking', Int. J. Eng. Comput. Sci., vol. 4, no. 3, pp. 11109-11112, 2015.

[17] N. Aherrahrou and H. Tairi, 'PDE based scheme for multi-modal medical image watermarking', Int. J. Biomed. Eng., vol. 14, pp. 108-126, 2015.

[18] L. Basyoni, H. I. Saleh, and M. B. Abdelhalim, 'Enhanced Watermarking Scheme for 3D Mesh Models', in 7th International Conference on Information Technology, 2015, pp. 612-619.

[19] W. Peng, X. Hongling, L. Wenlin, and S. Wenlong, 'Harris Scale Invariant Corner Detection Algorithm Based on the Significant Region', Int. J. Signal Process. Image Process. Pattern Recognit., vol. 9, no. 3, pp. 413-420, 2016.

[20] 'Robust Formal Watermarking Model Based on the Hyperbolic Geometry for Image Security', Int. J. Recent Technol. Eng., vol. 9, no. 3, pp. 617-629, Sep. 2020, doi: 10.35940/ijrte.C4651.099320.

[21] '(PDF) An Analysis and Implementation of the Harris Corner Detector'. https://www.researchgate.net/publication/328050891_An_Analysis_and_Implementation_of_the_Har ris_Corner_Detector (accessed Jun. 16, 2021).

[22] D. Hilbert, 'Foundations of Geometry', PhD Thesis, Univerty of Göttingen, 1950.

[23] M. P., 'Premiers principes de la MétagéométrieouGéométriegénérale', Rev. Néo-Scolastique 3ème Anné, vol. 1896, no. 10, pp. 143-170, 1896.

[24] T. S., Hyperbolic geometry: history, models, and axioms. 2004.

[25] D. T. Tiendrebeogo, 'CheickAmedDiloma Gabriel Traore', p. 65.

[26] M. A., Unafficheurgénériqued'arbres à l’aide de la géométriehyperbolique. 2000.

[27] A. F. Beardon and D. Minda, 'The hyperbolic metric and geometric function theory', 2006.

[28] S. L., 'A catadioptric sensor with multiple viewpoints', Robot. Auton. Syst., vol. 51, pp. 667-674, 2005.

[29] B. S. and N. S. K., 'A theory of single-viewpoint catadioptric image formation', Int. J. Comput. Vis., vol. 35, no. 2, pp. 1-22, 1999.

[30] B. D. A. et al., Geometry. 2002. [Online]. Available: http://www.cut-theknot.org/ctk/Parabola.shtml\#Brannan

[31] '(PDF) Combined DWT-DCT digital image watermarking'. https://www.researchgate.net/publication/26621646_Combined_DWT-

DCT_digital_image_watermarking?enrichId=rgreq-9434c057cfb4d8b92186b0e733e2c214XXX\&enrichSource $=Y 292 Z X J Q Y W d 1 O z I 2 N j I x N j Q 2 O 0 F T O j E 1 M z I y N D I x M j M 4 O T g 5 M k A x N D E$ zNTQyOTA3OTQy\&el=1_x_3\&_esc=publicationCoverPdf (accessed Jul. 07, 2021).

[32] 'An improved secure image hiding technique using PN-sequence based on DCT-OTP | IEEE Conference Publication | IEEE Xplore'. https://ieeexplore.ieee.org/abstract/document/8276336 (accessed Jun. 16, 2021).

\section{AuTHORS}

Cheick Yacouba Rachid Coulibaly I have a master's degree from Nazi Boni University in information systems and decision support systems. My interest research topic is image watermarking for decision supportand geolocation system .

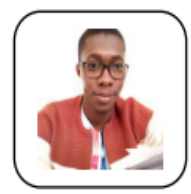

Telesphore Tiendrebeogo $\mathrm{PhD}$ and overlay network and assistant professor at Nazi Boni University. I have a master's degree in multimedia andreal time system. My current research is on big data and image watermarking.

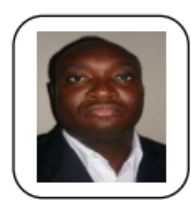

